\title{
Irreversible Investment Problems
}

\author{
Anders Øksendal \\ Department of Mathematics \\ University of Oslo \\ P.O.Box 1053 Blindern \\ $\mathrm{N}-0316$ Oslo \\ Norway \\ E-mail: anderso@math.uio.no
}

12th June 1998

This paper mathematically treats the following economic problem: A company wants to expand its capacity in investments that are irrevesible. The problem is to find the best investment strategy taking the fluctuating market into account. We give some implicit conditions for a solution in the case where the market process is n-dimensional and an explicit solution and existence of a solution in the case where the market process is one dimensional.

KEY WORDS Optimal stochastic control, irreversible investments, monotone increasing controls, Feller processes

\section{Introduction}

This paper focuses on the problem of investing in an uncertain market, when the investments are considered to be irreversible. This means that once an investment has been made and the market later drops to a less favorable state, we cannot undo the investment. The risk of overinvesting means that we should wait longer to invest, than if the investments were totally or partially reversible (see [1]). On the other hand we do not want to wait too long and miss out on any profits due to our lack of capacity. The problem then is to find a proper investment strategy taking the fluctuating marked into account. Numerous examples of irreversible investments exist, for example purchase of highly specified production machinery, educating staff members or spending money on advertising. See Dixit and Pindyck [3] for further economic discussions of the problem.

We assume that our investments have two effects on our economy. The first is that the income will increase. In general the income will depend on the current state of the market and the current investment level, which will be denoted by $\theta$ and $k$, respectively. This is reasonable since a favourable market could for instance mean greater sales of a product. On the other hand a high capacity could result in higher maintenance costs. The income function will be denoted by

$$
\Pi(\theta, k): E \times[0, \infty) \rightarrow R
$$

where $\mathrm{E}$ denotes the set of all possible states of the market process.

The other effect of an investment is obviously that it costs money. We will assume that an investment has different costs depending on what our capacity is and naturally 
also depending on how much we want to increase our capacity with. However in general we could also have that the price of an investment is dependent on the market situation. For example a good market could call for a greater demand in production machinery, thus raising the price of such equipment. The function $\Gamma(\theta, k)$ will be such that an increase in capacity from $k$ to $q$, when the market is at the state $\theta$, will cost

$$
\Gamma(\theta, q)-\Gamma(\theta, k)
$$

In addition we have a discount factor $\lambda$ built into the model. This factor is considered to be strictly positive. We also consider the discount factor to be constant.

Another assumption is that the market process is not affected by the investments made. This is a valid assumption if we are considered to be small investors in a large market.

The market process will be denoted by $\Theta_{t}$. It takes values in the set $E \subseteq \mathbf{R}^{n}$. We will show that the solution to the irreversible investment problem is to find a forbidden region $\mathcal{F} \subseteq E \times[0, \infty)$, such that the optimal control is a vertical reflection off this set. This means that it is optimal to invest whenever the pair $(\theta, k)$ is in $\mathcal{F}$ and also to increase our capacity $k$ such that $(\theta, k)$ no longer is in $\mathcal{F}$.

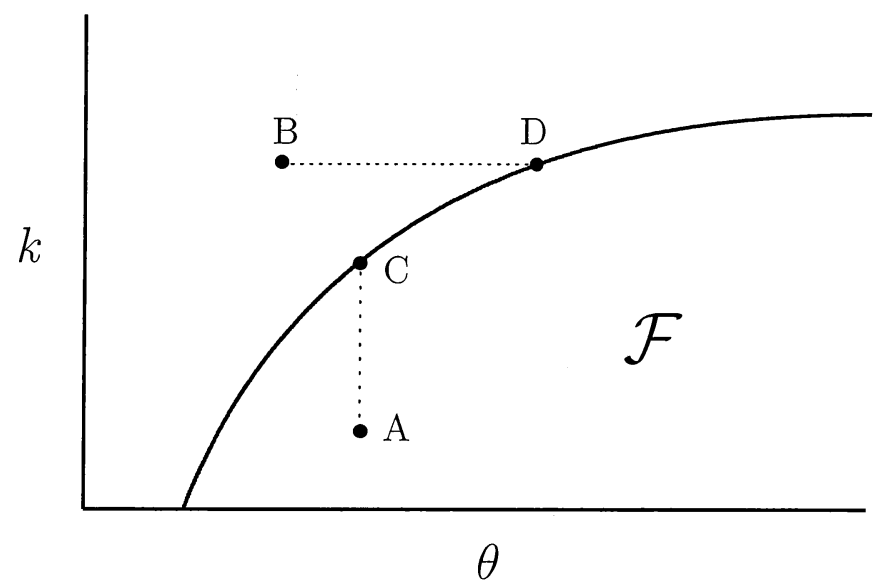

This situation can be seen in the figure above, where we consider a one dimensional market process. Suppose we are in the state $A$. Here the market is in a relatively good state, but our production capacity is low. We see that the point $A$ is in the forbidden region and therefore we should invest. We also see that we should immedeately increase our capacity $k$ until we hit the boundary of the forbidden region (point $C$ ). If we on the other hand start outside the forbidden region, for example at the point $B$, where the market is less profitable and the capacity is high. Then we should not invest immedeately, but wait until the marked changes into a more favourable state. More precisely the marked be such that the pair $(\theta, k)$ reaches $D$ until we start investing again, and then we should invest just enough to keep us out of the forbidden region.

Later it will also be shown that in order to find the forbidden region, we need only know when (and not how much) to start investing for each investment level, then we can use this to construct a forbidden region. In the paper this corresponds to finding $k$-crossections of the forbidden region, which will be called forbidden candidates (denoted by $\mathcal{F}_{k}$ ). Essentially we will show that if this family of sets is decreasing in $k$, then this family of sets gives the forbidden region $\mathcal{F}$ that solves the irreversible investment problem.

This paper is organized as follows. Section 2 introduces some preliminary results needed in order to make the theory work. These results are quite well known, allthough possibly 
not in the exact form given here. Here we also give conditions on the controls, marked process and functions $\Pi$ and $\Gamma$. In section 2.4 we will present the precise mathematical interpretation of the problem. In section 3.1 sufficient conditions for a solution are given. These conditions will be rather intricate and in section 3.2 they are narrowed down to conditions on the forbidden area $\mathcal{F}$. Section 4 gives the existence of and an explicit solution to the problem in a simplified one dimensional case.

This paper generalizes the result in T.Ø.Kobilas [7] article on irreversible investment problems, where a similar result is shown for the case where the market process is a geometric brownian motion. The proofs in Kobilas paper mainly rely on stochastic integration in order to make things work. This means that the processes we work with should be Ito processes or at least semi-martingales if the generalization in Protter [8] is used. This paper does not use this approach, but uses theory developed by Dynkin [4] to solve the problem. The paper also provides some genearalization to finding the specific solution to the irreversible investment problem.

This paper is based on my Cand.Scient.thesis in mathematics at the University of Oslo. My supervisor has been Tom Lindstrøm. I would like to thank him for his help and patience with my questions and ideas during the last year, and also for introducing me to the irreversible investment problem, which has been interesting to work with. I would also like to thank him for reading through the many versions of this paper. 


\section{Preliminary results}

\subsection{The Market Process}

The market process, denoted by $\Theta_{t}$, is assumed to be a time-homogeneous strong. Feller process on the state space $E$. This means that the function

$$
u(\theta, t)=E^{\theta}\left[f\left(\Theta_{t}\right)\right]
$$

is a continuous function of $(\theta, t)$ for every measurable bounded function $f(\theta)$.

\subsection{The Controls}

We will let functions of the form

$$
K_{t}(\omega):[0, \infty) \times \Omega \rightarrow[0, \infty)
$$

represent the investment strategies. We want these control functions $K_{t}(\omega)$ to be increasing as a function of time. In addition we will need to integrate the control function with respect to $t$ and $\omega$ so we will need a measurability requirement. Another assumption on the control is that the decision on whether to invest or not should be independent on the market price at a later stage, because we don't know for sure what the future market price will be. This condition can be stated mathematically using adaptedness of the control function to the $\sigma$-algebra generated by the market process. A last condition is that the process is right-continuous. This result is needed for the process to be properly approximated by controls that only have finite jumps and otherwise are constant. More precisely the control functions $K_{t}(\omega)$ will be considered to be:

1. Measurable with respect to the $\sigma$-algebra $\mathcal{B}_{[0, \infty)} \times \mathcal{M}$

2. Non-decreasing as a function of $t$, for a.e. $\omega$

3. Right-continuous as a function of $t$, for a.e. $\omega$

4. Adapted to the filtration $\mathcal{M}_{t}=\left\{\sigma\left(\Theta_{s}\right) ; s \leq t\right\}$

Control functions satisfying these four conditions and starting at the investment capacity above or equal to $k$ and the market beginning in the state $\theta$ will be called $(\theta, k)$ strategies.

\subsection{Some useful results}

Before the precise mathematical problem is presented, some results are needed. The resolvent of a function $f$ will sometimes be denoted by $R_{\lambda} f$ :

$$
R_{\lambda} f(\theta)=E^{\theta}\left[\int_{0}^{\infty} e^{-\lambda t} f\left(\Theta_{t}\right) d t\right]
$$

Here we will assume that $\lambda$ is a strictly positive constant. The following theorem will be used throughout this entire paper. It can be found in Dynkin's book, Markov processes [4], where the only restriction on the functions is that the integrals converge absolutely:

Theorem 2.1 (Dynkin's theorem). Let $\Theta_{t}$ be a strongly measurable strong Markov process, and let $\tau$ be a Markov time for $\Theta_{t}$. If $f=R_{\lambda} h$ then

$$
E^{\theta}\left[e^{-\lambda \tau} f\left(\Theta_{\tau}\right)\right]-f(\theta)=-E^{\theta}\left[\int_{0}^{\tau} e^{-\lambda t} h\left(\Theta_{t}\right) d t\right]
$$


Proof. See Dynkin [4] pg.132 (Theorem 5.1) for the proof.

In the following theorems $\mathcal{G}$ denotes the set of all bounded measurable functions $f$, such that $E^{\theta}\left[f\left(\Theta_{t}\right)\right]$ converges to $f(\theta)$ as $t \rightarrow 0$, for every $\theta$. And $\mathcal{D}(A, \mathcal{G})$ denotes the subset of $\mathcal{G}$ for which the generator exists.

Lemma 2.2. The set of all bounded continuous functions is a subset of $\mathcal{G}$.

Proof. The dominated convergence theorem gives

$$
\lim _{t \rightarrow 0} E^{\theta}\left[f\left(\Theta_{t}\right)\right]=E^{\theta}\left[\lim _{t \rightarrow 0} f\left(\Theta_{t}\right)\right]=E^{\theta}\left[f\left(\Theta_{0}\right)\right]=f(\theta)
$$

There is an important connection between the resolvent and the generator of a function, the following result can be found in Breiman [2].

Theorem 2.3. 1. If $f \in \mathcal{G}$, then $R_{\lambda} f$ is in $\mathcal{D}(A, \mathcal{G})$ and

$$
(\lambda-A)\left(R_{\lambda} f\right)=f
$$

2. If $f$ is in $\mathcal{D}(A, \mathcal{G})$, then

$$
R_{\lambda}((\lambda-A) f)=f
$$

Proof. See Breiman [2] (pg.342) for the proof.

The theorem has an important application which will be used later to find an explicit expression for the resolvent.

Corollary 2.4. If $f$ is a bounded continuous function then the equation

$$
A u-\lambda u=-f
$$

has one and only one bounded solution in $\mathcal{D}(A, \mathcal{G})$, and this solution is $u(\theta)=R_{\lambda} f(\theta)$.

Proof. $f(\theta)$ is bounded and continuous and therefore in $\mathcal{G}$. Assume that $u(\theta)$ is a bounded function in $\mathcal{D}(A, \mathcal{G})$ and that $u(\theta)$ satisfies the equation

$$
A u-\lambda u=-f
$$

Then taking the resolvent on each side gives

$$
R_{\lambda}(A u-\lambda u)=-R_{\lambda} f
$$

Now since $u(\theta) \in \mathcal{D}(A, \mathcal{G})$ and the left side equals $-u(\theta)$ by theorem 2.3, we have

$$
u(\theta)=R_{\lambda} f
$$

The following definition gives the set of functions which we will work with in this paper. 
Definintion 2.5. $L_{\Theta}^{1}$ is the set of all measurable functions $f: E \rightarrow R$ such that:

$$
R_{\lambda}|f|(\theta)=E^{\theta}\left[\int_{0}^{\infty} e^{-\lambda t}\left|f\left(\Theta_{t}\right)\right| d t\right]
$$

is bounded for $\theta \in E$

The next theorem gives a different version of theorem 2.1 (Dynkin's theorem), which will be used several times later. A similar result can also be found in Dynkin [4] pg.133.

Theorem 2.6 (Dynkin's formula). Suppose $f$ is a bounded continuous function in $\mathcal{D}(A)$ such that $A f-\lambda f \in L_{\Theta}^{1}$. Let $\tau$ denote a stopping time, then

$$
E^{\theta}\left[e^{-\lambda \tau} f\left(\Theta_{\tau}\right)\right]-f(\theta)=E^{\theta}\left[\int_{0}^{\tau} e^{-\lambda t}(A f-\lambda f)\left(\Theta_{t}\right) d t\right]
$$

Proof. By lemma 2.2 and theorem 2.3, then

$$
E^{\theta}\left[e^{-\lambda \tau} f\left(\Theta_{\tau}\right)\right]-f(\theta)=E^{\theta}\left[e^{-\lambda \tau} R_{\lambda}(\lambda f-A f)\left(\Theta_{\tau}\right)\right]-R_{\lambda}(\lambda f-A f)(\theta)
$$

Using theorem 2.1, this equals

$$
E^{\theta}\left[\int_{0}^{\tau} e^{-\lambda t}(A f-\lambda f)\left(\Theta_{t}\right) d t\right]
$$

It turns out that all the $(\theta, k)$-strategies can be approximated pointwise by functions that only have a finite amount of jumps and otherwise are constant.

Proposition 2.7. Every $(\theta, k)$-strategy can be written as a pointwise limit of simple functions of the form

$$
K_{t}^{n}=\sum_{i=0}^{10^{2 n}} k_{i}^{n} \chi_{\left\{\tau_{i}(\omega) \leq t<\tau_{i+1}(w)\right\}}
$$

where $\left\{k_{i}^{n}\right\}$ denotes a partition of the interval $\left[k, k+10^{n}\right]$ such that

$$
k_{i}^{n}=k+i \cdot 10^{-n} \quad, \quad i=0,1,2,3, \ldots, 10^{2 n}+1
$$

and $\tau_{i}$ denotes the first hitting time for the process $K_{t}$ to the interval $\left[k_{i}, \infty\right)$ whenever $i \leq 10^{2 n}$. We will let $\tau_{10^{2 n}+1}=\infty$.

Proof. (Outlined)

For each $(t, \omega)$ then there exists $n, i \in \mathbf{N}$ such that

$$
k_{i}^{n} \leq K_{t}<k_{i+1}^{n}
$$

But then we only need to show that that this implies $t \in\left[\tau_{i}(\omega), \tau_{i+1}(\omega)\right)$ But then $K_{t}^{n} \leq$ $K_{t}<K_{t}^{n}+10^{-n}$. Details are left to the reader. For a full proof see [9].

This is useful since a lot of results are easier to prove for such simple functions. The results can then be generalized to all $(\theta, k)$-strategies by using the limit theorems. Note also that we have that

$$
K_{t}^{n, a}(\omega) \leq K_{t}^{n+1, a}(\omega) \leq K_{t}(\omega)
$$

In other words the controls are increasing in $n$. 


\subsection{Conditions}

In order to make the theory work, some conditions on the functions $\Pi$ and $\Gamma$ are needed. Basically these conditions are needed for the limit theorems to hold. We also need some conditions that make it possible to apply Dynkin's theorem and Dynkin's formula.

\section{Conditions 2.8.}

1. $\Gamma$ is continuous and bounded in $\theta$ for every $k$ and non-decreasing in the $k$ variable. $A \Gamma-\lambda \Gamma$ exists, and is continuous, continuously differentiable wrt. $k$, and in $L_{\Theta}^{1}$ for every $k$.

2. $\Pi$ is non-negative, continuous and continuously differentiable wrt. $k$.

3. The function $S^{+}(\theta)=\max \{0, \sup \{(\Pi+A \Gamma-\lambda \Gamma)(\theta, k): k \geq 0\}\}$ is in $L_{\Theta}^{1}$.

4. The functions

$$
M^{(q)}(\theta)=\sup \{|(\Pi+A \Gamma-\lambda \Gamma)(\theta, k)|: 0 \leq k \leq q\}
$$

and

$$
N^{(q)}(\theta)=\sup \left\{\left|\left(\Pi_{k}+A \Gamma_{k}-\lambda \Gamma_{k}\right)(\theta, k)\right|: 0 \leq k \leq q\right\}
$$

are in $L_{\Theta}^{1}$ for all $q \in[0, \infty)$.

5. The function $\left(\Pi_{k}+A \Gamma_{k}-\lambda \Gamma_{k}\right)(\theta, k)$ is strictly decreasing in the $k$-variable.

6. $\left(\Pi_{k}+A \Gamma_{k}-\lambda \Gamma_{k}\right)(\theta, k)$ is bounded in $\theta$ for every $k$.

The fifth condition regulates the behaviour of $(\Pi+A \Gamma-\lambda \Gamma)(\theta, k)$ such that it is possible to prove the following limit theorem quite easily.

Lemma 2.9. Suppose $\Pi$ and $\Gamma$ satisfy conditions 2.8 and let $K_{t}$ be any $(\theta, k)$-strategy, and if $\left\{K_{t}^{n}\right\}$ denotes an increasing family of controls such that $K_{t}^{n} \rightarrow K_{t}$ as $n \rightarrow \infty$. Then

$$
\begin{aligned}
& E^{\theta}\left[\int_{0}^{\infty} e^{-\lambda t}(\Pi+A \Gamma-\lambda \Gamma)\left(\Theta_{t}, K_{t}\right) d t\right] \\
= & \lim _{n \rightarrow \infty} E^{\theta}\left[\int_{0}^{\infty} e^{-\lambda t}(\Pi+A \Gamma-\lambda \Gamma)\left(\Theta_{t}, K_{t}^{n}\right) d t\right]
\end{aligned}
$$

Proof. (Outlined)

Let $P=\left\{(\theta, k):\left(\Pi_{k}+A \Gamma_{k}-\lambda \Gamma_{k}\right)(\theta, k) \geq 0\right\}$ and $N=P^{C}$. The proof concists of taking the limit as $n \rightarrow \infty$ of the two functions

$\lim _{n \rightarrow \infty} E^{\theta}\left[\int_{0}^{\infty} e^{-\lambda t}(\Pi+A \Gamma-\lambda \Gamma) \chi_{P}\left(\Theta_{t}, K_{t}^{n}\right) d t\right]+E^{\theta}\left[\int_{0}^{\infty} e^{-\lambda t}(\Pi+A \Gamma-\lambda \Gamma) \chi_{N}\left(\Theta_{t}, K_{t}\right) d t\right]$

Then the result follows by the dominated convergence theorem (using $S^{+}+\mid(\Pi+A \Gamma-$ $\lambda \Gamma)\left(\theta, k_{0}\right) \mid$ as a bound function) on the first expectation and by monotone convergence on the second. See [9] for a complete proof. 


\subsection{The mathematical problem}

Now we are ready to present the precise mathematical problem. We want to maximize the expected profit after we have deducted the expected cost of our investments. As in the Kobila article it can be shown that this can be expressed using the resolvent of the function $(\Pi+A \Gamma-\lambda \Gamma)(\theta, k)$. To see this first note that the total expected discounted income is given by

$$
E^{\theta}\left[\int_{0}^{\infty} e^{-\lambda t} \Pi\left(\Theta_{t}, K_{t}\right) d t\right]
$$

where $\Pi(\theta, k)$ denotes the income rate when the market is in the state $\theta$ and our investment level is $k$.

The expected reduction of funds due to the investments requires more work. Here first assume that the $(\theta, k)$-strategy is a simple control of the form given in proposition 2.7. Fix an $\omega_{0} \in \Omega$. Then for each jump, say from $k_{i-1}$ to $k_{i}$, the increase in capacity is made at the time $\tau_{i}\left(\omega_{0}\right)$. The cost of this capacity increase is $e^{-\lambda \tau_{i}\left(\omega_{0}\right)}\left[\Gamma\left(\Theta_{\tau_{i}}\left(\omega_{0}\right), k_{i}\right)-\Gamma\left(\Theta_{\tau_{i}}\left(\omega_{0}\right), k_{i-1}\right)\right]$ and the expected cost is therefore

$$
E^{\theta}\left[e^{-\lambda \tau_{i}}\left[\Gamma\left(\Theta_{\tau_{i}}, k_{i}\right)-\Gamma\left(\Theta_{\tau_{i}}, k_{i-1}\right)\right]\right]
$$

The cost of all the investments is naturally given by the sum of such investments

$$
\sum_{i=1}^{N} E^{\theta}\left[e^{-\lambda \tau_{i}}\left[\Gamma\left(\Theta_{\tau_{i}}, k_{i}\right)-\Gamma\left(\Theta_{\tau_{i}}, k_{i-1}\right)\right]\right]
$$

This sum can be rewritten as

$$
\begin{aligned}
= & \sum_{i=1}^{N} E^{\theta}\left[e^{-\lambda \tau_{i}} \Gamma\left(\Theta_{\tau_{i}}, k_{i}\right)\right]-\sum_{i=1}^{N} E^{\theta}\left[e^{-\lambda \tau_{i}} \Gamma\left(\Theta_{\tau_{i}}, k_{i-1}\right)\right] \\
& =E^{\theta}\left[e^{-\lambda \tau_{N}} \Gamma\left(\Theta_{\tau_{N}}, k_{N}\right)\right]+\sum_{i=0}^{N-1} E^{\theta}\left[e^{-\lambda \tau_{i}} \Gamma\left(\Theta_{\tau_{i}}, k_{i}\right)\right] \\
& -\sum_{i=0}^{N-1} E^{\theta}\left[e^{-\lambda \tau_{i+1}} \Gamma\left(\Theta_{\tau_{i+1}}, k_{i}\right)\right]-E^{\theta}\left[e^{-\lambda \tau_{0}} \Gamma\left(\Theta_{\tau_{0}}, k_{0}\right)\right]
\end{aligned}
$$

Recall that the controls are adapted to the process $\Theta_{t}$, so therefore all $K_{t}$-stopping times are also $\Theta_{t}$-stopping times. Then Dynkin's formula can be applied. It has the form

$$
E^{\theta}\left[e^{-\lambda \tau} \Gamma\left(\Theta_{\tau}, k\right)\right]-\Gamma(\theta, k)=E^{\theta}\left[\int_{0}^{\tau} e^{-\lambda t}(A \Gamma-\lambda \Gamma)\left(\Theta_{t}, k\right) d t\right]
$$

which again means that for stopping times $\tau_{1}$ and $\tau_{2}$, then

$$
E^{\theta}\left[e^{-\lambda \tau_{2}} \Gamma\left(\Theta_{\tau_{2}}, k\right)\right]-E^{\theta}\left[e^{-\lambda \tau_{1}} \Gamma\left(\Theta_{\tau_{1}}, k\right)\right]=E^{\theta}\left[\int_{\tau_{1}}^{\tau_{2}} e^{-\lambda t}(A \Gamma-\lambda \Gamma)\left(\Theta_{t}, k\right) d t\right]
$$

for arbitrary $k \in[0, \infty)$. Using this in $\left(^{*}\right)$ then gives

$$
=-E^{\theta}\left[\int_{\tau_{N}}^{\infty} e^{-\lambda t}(A \Gamma-\lambda \Gamma)\left(\Theta_{t}, k_{N}\right) d t\right]-\sum_{i=0}^{N-1} E^{\theta}\left[\int_{\tau_{i}}^{\tau_{i+1}} e^{-\lambda t}(A \Gamma-\lambda \Gamma)\left(\Theta_{t}, k_{i}\right) d t\right]
$$




$$
-\Gamma\left(\theta, k_{0}\right)
$$

If we denote the simple control by $K_{t}^{N}$, then this expression equals

$$
=-E^{\theta}\left[\int_{0}^{\infty} e^{-\lambda t}(A \Gamma-\lambda \Gamma)\left(\Theta_{t}, K_{t}^{N}\right) d t\right]-\Gamma(\theta, k)
$$

Then we have an expression for the expected cost of capacity increase with the simple controls. Then for all simple controls the expected profit is given by

$$
E^{\theta}\left[\int_{0}^{\infty} e^{-\lambda t}(\Pi+A \Gamma-\lambda \Gamma)\left(\Theta_{t}, K_{t}^{N}\right) d t\right]+\Gamma(\theta, k)
$$

So what happends if the control is not simple? Let $K_{t}$ denote a $(\theta, k)$-strategy. Then according to proposition 2.7 there exists a sequence of $(\theta, k)$-strategies $\left\{K_{t}^{n}\right\}_{n=1}^{\infty}$ such that $K_{t}^{n} \rightarrow K_{t}$ as $n \rightarrow \infty$. For each of the simple controls the total profit is given by

$$
\begin{gathered}
E^{\theta}\left[\int_{0}^{\infty} e^{-\lambda t} \Pi\left(\Theta_{t}, K_{t}^{n}\right) d t\right]+\sum_{i=1}^{10^{2 n}} E^{\theta}\left[e^{-\lambda \tau_{i}}\left[\Gamma\left(\Theta_{\tau_{i}}, k_{i}\right)-\Gamma\left(\Theta_{\tau_{i}}, k_{i-1}\right)\right]\right] \\
=E^{\theta}\left[\int_{0}^{\infty} e^{-\lambda t}(\Pi+A \Gamma-\lambda \Gamma)\left(\Theta_{t}, K_{t}^{n}\right) d t\right]+\Gamma(\theta, k)
\end{gathered}
$$

Therefore since the controls are increasing in $n$, lemma 2.9 gives that the expected profit converges as $n \rightarrow \infty$ and converges to

$$
+E^{\theta}\left[\int_{0}^{\infty} e^{-\lambda t}(\Pi+A \Gamma-\lambda \Gamma)\left(\Theta_{t}, K_{t}\right) d t\right]+\Gamma(\theta, k)
$$

This is the expression for the expected cost of the investments that will be used in this paper. But the expression $\Gamma(\theta, k)$ is not dependent on the control $K_{t}$, so if we let

$$
J^{(\theta, k)}\left(K_{t}\right)=E^{\theta}\left[\int_{0}^{\infty} e^{-\lambda t}(\Pi+A \Gamma-\lambda \Gamma)\left(\Theta_{t}, K_{t}\right) d t\right]
$$

the problem is to find the $(\theta, k)$-strategy that maximizes this expression. Or in other words finding a $(\theta, k)$-strategy $K_{t}^{*}$ such that

$$
J^{(\theta, k)}\left(K_{t}^{*}\right)=\sup \left\{J^{(\theta, k)}\left(K_{t}\right): K_{t}(\theta, k) \text {-strategies }\right\}
$$

We also want to know what the maximum value $J^{(\theta, k)}\left(K_{t}^{*}\right)$ is. 


\section{Constructing a solution}

\subsection{Sufficient conditions}

In this section the process $\Theta_{t}$ is considered to be n-dimensional, unless otherwise specified. The assumptions on the process are as in section 2.1 and the conditions on the functions $\Pi$ and $\Gamma$ are as in conditions 2.8. Using this we can give sufficient conditions for a solution to the problem. A similar proposition can be found in the article by Kobila [7].

Proposition 3.1. Assume there exists a set $\mathcal{F}$ such that:

1. $\phi(\theta)=\sup \{k:(\theta, k) \in \mathcal{F}\}$ is continuous.

2. $\left(\Pi_{k}+A \Gamma_{k}-\lambda \Gamma_{k}\right)(\theta, k) \geq 0$, for all $(\theta, k) \in \mathcal{F}$.

3. Suppose

$$
\nu(\theta, k)=E^{\theta}\left[\int_{0}^{\infty} e^{-\lambda t} H\left(\Theta_{t}, k\right) d t\right]
$$

where

$$
H(\theta, k)=\left\{\begin{array}{lr}
(\Pi+A \Gamma-\lambda \Gamma)(\theta, k) & \text { when }(\theta, k) \notin \mathcal{F} \\
(\Pi+A \Gamma-\lambda \Gamma)(\theta, \phi(\theta)) & \text { when }(\theta, k) \in \mathcal{F}
\end{array}\right.
$$

then $\nu(\theta, k)$ is bounded in $\theta$ for every $k$.

4. The partial derivative $\nu_{k}(\theta, k)$ exists and

$$
\nu_{k}(\theta, k)\left\{\begin{array}{l}
\leq 0 \text { when }(\theta, k) \notin \mathcal{F} \\
=0 \text { when }(\theta, k) \in \mathcal{F}
\end{array}\right.
$$

Then for all $(\theta, k)$

$$
\sup _{K_{t}} E^{(\theta, k)}\left[\int_{0}^{\infty} e^{-\lambda t}(\Pi+A \Gamma-\lambda \Gamma)\left(\Theta_{t}, K_{t}\right) d t\right]=\nu(\theta, k)
$$

where the supremum is taken over all $(\theta, k)$-strategies. Equality holds for

$$
K_{t}^{*}=k \vee \sup \left\{\phi\left(\Theta_{s}\right) ; s \leq t\right\}
$$

Proof. As before rewriting Dynkin's theorem 2.1 for stopping times $\tau_{1}$ and $\tau_{2}$ gives

$$
E^{\theta}\left[e^{-\lambda \tau_{2}} \nu\left(\Theta_{\tau_{2}}, k\right)\right]-E^{\theta}\left[e^{-\lambda \tau_{1}} \nu\left(\Theta_{\tau_{1}}, k\right)\right]=-E^{\theta}\left[\int_{\tau_{1}}^{\tau_{2}} e^{-\lambda t} H\left(\Theta_{t}, k\right) d t\right]
$$

for arbitrary $k \in[0, \infty)$. Now let $K_{t}$ be any $(\theta, k)$-strategy, then it can be approximated by simple controls $\left\{K_{t}^{n}\right\}$ of the form given in proposition 2.7

$$
K_{t}^{n}=\sum_{i=0}^{10^{2 n}} k_{i}^{n} \chi_{\left\{\tau_{i}(\omega) \leq t<\tau_{i+1}(\omega)\right\}}
$$

where $\tau_{i}$ denotes $K_{t}$-stopping times (and setting $N=10^{2 n}$ for simplicity of notation). But for all such simple controls

$$
E^{\theta}\left[\int_{0}^{\infty} e^{-\lambda t} H\left(\Theta_{t}, K_{t}^{n}\right) d t\right]
$$




$$
=E^{\theta}\left[\sum_{i=0}^{N-1} \int_{\tau_{i}}^{\tau_{i+1}} e^{-\lambda t} H\left(\Theta_{t}, K_{t}^{n}\right) d t\right]+E^{\theta}\left[\int_{\tau_{N}}^{\infty} e^{-\lambda t} e^{-\lambda t} H\left(\Theta_{t}, K_{t}^{n}\right) d t\right]
$$

Whenever $t \in\left[\tau_{i}\left(\omega_{0}\right), \tau_{i+1}\left(\omega_{0}\right)\right)$ the control $K_{t}^{n}\left(\omega_{0}\right)$ is constant and equal to $k_{i}$.

$$
=\sum_{i=0}^{N-1} E^{\theta}\left[\int_{\tau_{i}}^{\tau_{i+1}} e^{-\lambda t} H\left(\Theta_{t}, k_{i}^{n}\right) d t\right]+E^{\theta}\left[\int_{\tau_{N}}^{\infty} e^{-\lambda t} H\left(\Theta_{t}, k_{N}^{n}\right) d t\right]
$$

$\tau_{i}$ is a $K_{t}$-stopping time, but since $K_{t}$ is adapted to the filtration generated by $\Theta_{t}$, then it is a $\Theta_{t}$-stopping time as well. Dynkin's formula then gives that the expression above equals

$$
\begin{gathered}
=\sum_{i=0}^{N-1} E^{\theta}\left[e^{-\lambda \tau_{i}} \nu\left(\Theta_{\tau_{i}}, k_{i}^{n}\right)\right]-\sum_{i=0}^{N-1} E^{\theta}\left[e^{-\lambda \tau_{i+1}} \nu\left(\Theta_{\tau_{i+1}}, k_{i}^{n}\right)\right]+E^{\theta}\left[e^{-\lambda \tau_{N}} \nu\left(\Theta_{\tau_{N}}, k_{N}^{n}\right)\right] \\
=\sum_{i=1}^{N} E^{\theta}\left[e^{-\lambda \tau_{i}} \nu\left(\Theta_{\tau_{i}}, k_{i}^{n}\right)-e^{-\lambda \tau_{i}} \nu\left(\Theta_{\tau_{i}}, k_{i-1}^{n}\right)\right]+\nu(\theta, k) \\
=\sum_{i=1}^{N} E^{\theta}\left[e^{-\lambda \tau_{i}}\left(\nu\left(\Theta_{\tau_{i}}, k_{i}^{n}\right)-\nu\left(\Theta_{\tau_{i}}, k_{i-1}^{n}\right)\right)\right]+\nu(\theta, k)
\end{gathered}
$$

Now $\nu_{k}(\theta, k) \leq 0$, for all $(\theta, k)$, and since $k_{i}^{n}>k_{i-1}^{n}$ then

$$
\nu\left(\Theta_{\tau_{i}}, k_{i}^{n}\right)-\nu\left(\Theta_{\tau_{i}}, k_{i-1}^{n}\right) \leq 0
$$

and therefore we also have that the expectation of this expression is negative, so we have that

$$
E^{\theta}\left[\int_{0}^{\infty} e^{-\lambda t} H\left(\Theta_{t}, K_{t}^{n}\right) d t\right] \leq \nu(\theta, k)
$$

for all $\mathrm{n}$. From the assumptions we have that $H(\theta, k)=(\Pi+A \Gamma-\lambda \Gamma)(\theta, \phi(\theta))$ whenever $(\theta, k) \in \mathcal{F}$, but since $\left(\Pi_{k}+A \Gamma_{k}-\lambda \Gamma_{k}\right)(\theta, k) \geq 0$ in $\mathcal{F}$, then $(\Pi+A \Gamma-\lambda \Gamma)(\theta, \phi(\theta)) \geq$ $(\Pi+A \Gamma-\lambda \Gamma)(\theta, k)$ in $\mathcal{F}$. This means that $H(\theta, k) \geq(\Pi+A \Gamma-\lambda \Gamma)(\theta, k)$ for all $(\theta, k)$. Then

$$
\nu(\theta, k) \geq E^{\theta}\left[\int_{0}^{\infty} e^{-\lambda t}(\Pi+A \Gamma+\lambda \Gamma)\left(\Theta_{t}, K_{t}^{n}\right) d t\right]
$$

for all $n$. Taking the limit as $n \rightarrow \infty$, and using lemma 2.9 we get

$$
\begin{gathered}
E^{\theta}\left[\int_{0}^{\infty} e^{-\lambda t}(\Pi+A \Gamma-\lambda \Gamma)\left(\Theta_{t}, K_{t}\right) d t\right] \\
=\lim _{n \rightarrow \infty} E^{\theta}\left[\int_{0}^{\infty} e^{-\lambda t}(\Pi+A \Gamma-\lambda \Gamma)\left(\Theta_{t}, K_{t}^{n}\right) d t\right] \leq \lim _{n \rightarrow \infty} \nu(\theta, k)=\nu(\theta, k)
\end{gathered}
$$

which shows the inequality. 
So let us show that the given control

$$
K_{t}^{*}=k \vee \sup \left\{\phi\left(\Theta_{s}\right): s \leq t\right\}
$$

in fact is optimal. Note that for all simple controls $K_{t}^{n}$ that invest only in the forbidden region $\mathcal{F}$, then we have as before

$$
E^{\theta}\left[\int_{0}^{\infty} e^{-\lambda t} H\left(\Theta_{t}, K_{t}^{n}\right) d t\right]=\sum_{i=1}^{N} E^{\theta}\left[e^{-\lambda \tau_{i}}\left(\nu\left(\Theta_{\tau_{i}}, k_{i}^{n}\right)-\nu\left(\Theta_{\tau_{i}}, k_{i-1}^{n}\right)\right)\right]+\nu(\theta, k)
$$

But since $\nu(\theta, k)$ is $k$-invariant in $\mathcal{F}$ the sum above equals $\nu(\theta, k)$. Suppose $\left\{K_{t}^{n}\right\}$ is a family of simple controls that converge to $K_{t}^{*}$ from below as $n \rightarrow \infty$. These controls only invest in the forbidden region, therefore

$$
\nu(\theta, k)=\lim _{n \rightarrow \infty} E^{\theta}\left[\int_{0}^{\infty} e^{-\lambda t} H\left(\Theta_{t}, K_{t}^{n}\right) d t\right]
$$

By the same arguments as in lemma 2.9 this equals

$$
E^{\theta}\left[\int_{0}^{\infty} e^{-\lambda t} H\left(\Theta_{t}, K_{t}^{*}\right) d t\right]
$$

But $K_{t}^{*}$ never enters $\mathcal{F}$, and since $H(\theta, k)=(\Pi+A \Gamma-\lambda \Gamma)(\theta, k)$ outside $\mathcal{F}$, this equals

$$
=E^{\theta}\left[\int_{0}^{\infty} e^{-\lambda t}(\Pi+A \Gamma-\lambda \Gamma)\left(\Theta_{t}, K_{t}^{*}\right) d t\right]
$$

This means that $K_{t}^{*}$ is optimal.

\subsection{Forbidden candidates}

Sufficient conditions for the problem are given in proposition 3.1. The object of this section is to simplify these conditions. It is seen that once the forbidden region $\mathcal{F}$ is given, we know by definition what the function $\phi(\theta)$ is. It also turns out that it is possible to give restrictions on the $k$-crossections of $\mathcal{F}$ such that the correspondingly defined functions $\phi(\theta)$ and $\nu(\theta, k)$ satisfy the conditions of proposition 3.1. The basic quality needed is that they are forbidden candidates, as we will define below.

Definintion 3.2. Let $U_{k_{0}}=\left\{\theta:\left(\Pi_{k}+A \Gamma_{k}-\lambda \Gamma_{k}\right)\left(\theta, k_{0}\right)<0\right\}$

Definintion 3.3. Given a $k_{0} \in[0, \infty)$. A Borel set $\mathcal{P}_{k_{0}}$ will be called a $k_{0}$-forbidden candidate if:

1. For all $\theta \in \mathcal{P}_{k_{0}}^{C}$,

$$
E^{\theta}\left[\int_{0}^{\infty} e^{-\lambda t}\left(\Pi_{k}+A \Gamma_{k}-\lambda \Gamma_{k}\right)\left(\Theta_{t}, k_{0}\right) \chi_{\mathcal{P}_{k_{0}}^{C}}\left(\Theta_{t}\right) d t\right] \leq 0
$$

2. For all $\theta \in \partial \mathcal{P}_{k_{0}}^{C}$,

$$
E^{\theta}\left[\int_{0}^{\infty} e^{-\lambda t}\left(\Pi_{k}+A \Gamma_{k}-\lambda \Gamma_{k}\right)\left(\Theta_{t}, k_{0}\right) \chi_{\mathcal{P}_{k_{0}}^{C}}\left(\Theta_{t}\right) d t\right]=0
$$


3. $U_{k_{0}} \subseteq \mathcal{P}_{k_{0}}^{C}$

Now we wish to show that solving the irreversible investment problem is really a matter of finding forbidden candidates. More precisely we want to show that if

1. $\mathcal{F}_{k}$ is a closed $k$-forbidden candidate for every $k \in[0, \infty)$

2. $\mathcal{F}_{q} \subseteq \mathcal{F}_{k}$, if $q>k$

3. If $\theta$ is a boundary point for $\mathcal{F}_{k}$ (except possibly on the boundary points of the state space), then $\theta \notin \mathcal{F}_{q}$ for any $q>k$.

then $\mathcal{F}=\left\{(\theta, k): \theta \in \mathcal{F}_{k}\right\}$ gives the forbidden region that solves the irreversible investment problem.

Lemma 3.4. $\phi(\theta)$ is a continuous function.

Proof. Note that

$$
\phi^{-1}\left(\left(k_{0}, \infty\right)\right)=\bigcup_{k>k_{0}} \mathcal{F}_{k}
$$

Assume that $\theta$ is a boundary point of $\cup_{k>k_{0}} \mathcal{F}_{k}$ and that $\theta \in \cup_{k>k_{0}} \mathcal{F}_{k}$. Then there exists a $q>k_{0}$ such that $\theta \in \mathcal{F}_{q}$. But then by the monotonicity of the forbidden candidates $\theta$ is a boundary point for $\mathcal{F}_{k}$ for all $k \in\left[k_{0}, q\right]$. This contradicts the third assumption on the family of forbidden candidates, so $\theta \notin \cup_{k>k_{0}} \mathcal{F}_{k}$. This means that $\cup_{k>k_{0}} \mathcal{F}_{k}$ is open and hence $\phi(\theta)$ is continuous.

Lemma 3.5. Suppose $\Theta_{t}$ is a strong Feller process on the state space E. Let $f(\theta)$ be a bounded function. Then the resolvent of $f(\theta)$ :

$$
R_{\lambda} f(\theta)=E^{\theta}\left[\int_{0}^{\infty} e^{-\lambda t} f\left(\Theta_{t}\right) d t\right]
$$

is continuous in the $\theta$-variable.

Proof. By bounded convergence and Fubini-Tonelli we have

$$
\lim _{\theta \rightarrow \theta_{0}} E^{\theta}\left[\int_{0}^{\infty} e^{-\lambda t} f\left(\Theta_{t}\right) d t\right]=\int_{0}^{\infty} e^{-\lambda t} \lim _{\theta \rightarrow \theta_{0}} E^{\theta}\left[f\left(\Theta_{t}\right) d t\right]
$$

using that $\Theta_{t}$ is a strong Feller process and Fubini-Tonelli again then gives

$$
\int_{0}^{\infty} e^{-\lambda t} E^{\theta_{0}}\left[f\left(\Theta_{t}\right)\right] d t=E^{\theta_{0}}\left[\int_{0}^{\infty} e^{-\lambda t} f\left(\Theta_{t}\right) d t\right]
$$

We are ready to start showing that the function $\nu(\theta, k)$ that we defined does in fact satisfy the conditions in proposition 3.1.

Now some properties of the forbidden candidates are needed in order to prove that $\nu(\theta, k)$ is differentiable wrt.k. 
Lemma 3.6. Let $k \in[0, \infty)$. If $F$ and $B$ are $k$-forbidden candidates and $F \subseteq B$. Suppose that $B$ is closed, then

$$
\begin{aligned}
& E^{\theta}\left[\int_{0}^{\infty} e^{-\lambda t}\left(\Pi_{k}+A \Gamma_{k}-\lambda \Gamma_{k}\right)\left(\Theta_{t}, k\right) \chi_{F^{C}}\left(\Theta_{t}\right) d t\right] \\
= & E^{\theta}\left[\int_{0}^{\infty} e^{-\lambda t}\left(\Pi_{k}+A \Gamma_{k}-\lambda \Gamma_{k}\right)\left(\Theta_{t}, k\right) \chi_{B^{C}}\left(\Theta_{t}\right) d t\right]
\end{aligned}
$$

Proof. If $\theta \in B$ then $\left(\Pi_{k}+A \Gamma_{k}-\lambda \Gamma_{k}\right)(\theta, k) \geq 0$ since $B$ is a k-forbidden candidate. But $\theta \in B$, so

$$
E^{\theta}\left[\int_{0}^{\infty} e^{-\lambda t}\left(\Pi_{k}+A \Gamma_{k}-\lambda \Gamma_{k}\right)\left(\Theta_{t}, k\right) \chi_{B^{C}}\left(\Theta_{t}\right) d t\right]=0
$$

and by the assumption that $F$ is a k-forbidden candidate we have that for all $\theta$

$$
E^{\theta}\left[\int_{0}^{\infty} e^{-\lambda t}\left(\Pi_{k}+A \Gamma_{k}-\lambda \Gamma_{k}\right)\left(\Theta_{t}, k\right) \chi_{F^{C}}\left(\Theta_{t}\right) d t\right] \leq 0
$$

But then

$$
\begin{aligned}
& E^{\theta}\left[\int_{0}^{\infty} e^{-\lambda t}\left(\Pi_{k}+A \Gamma_{k}-\lambda \Gamma_{k}\right)\left(\Theta_{t}, k\right) \chi_{F^{C}}\left(\Theta_{t}\right) d t\right] \\
= & E^{\theta}\left[\int_{0}^{\infty} e^{-\lambda t}\left(\Pi_{k}+A \Gamma_{k}-\lambda \Gamma_{k}\right)\left(\Theta_{t}, k\right) \chi_{F^{C} \backslash B^{C}}\left(\Theta_{t}\right) d t\right]
\end{aligned}
$$

The the first of these expectations is less than or equal to zero, by $\left(^{*}\right)$ and the last expectation is greater than or equal to zero since it is an integral over a positive function. Since they are equal, this means that they both must be zero. So for all $\theta \in B$ the equality holds.

Assume that $\theta \notin B$. Let $\tau_{B}$ denote the first hitting time for the process $\Theta_{t}$ to the set $B$. Then

$$
\begin{aligned}
& E^{\theta}\left[\int_{0}^{\infty} e^{-\lambda t}\left(\Pi_{k}+A \Gamma_{k}-\lambda \Gamma_{k}\right)\left(\Theta_{t}, k\right) \chi_{F^{C} \backslash B^{C}}\left(\Theta_{t}\right) d t\right] \\
= & E^{\theta}\left[\int_{0}^{\tau_{B}} e^{-\lambda t}\left(\Pi_{k}+A \Gamma_{k}-\lambda \Gamma_{k}\right)\left(\Theta_{t}, k\right) \chi_{F^{C} \backslash B^{C}}\left(\Theta_{t}\right) d t\right] \\
+ & E^{\theta}\left[\int_{\tau_{B}}^{\infty} e^{-\lambda t}\left(\Pi_{k}+A \Gamma_{k}-\lambda \Gamma_{k}\right)\left(\Theta_{t}, k\right) \chi_{F^{C} \backslash B^{C}}\left(\Theta_{t}\right) d t\right]
\end{aligned}
$$

The first integral is zero since $\Theta_{t}(\omega) \notin F^{C} \backslash B^{C}$, whenever $0 \leq t \leq \tau_{B}(\omega)$. By Dynkin's theorem 2.1, the second integral equals

$$
E^{\theta}\left[e^{-\lambda \tau_{B}} E^{\Theta_{\tau_{B}}}\left[\int_{0}^{\infty} e^{-\lambda t}\left(\Pi_{k}+A \Gamma_{k}-\lambda \Gamma_{k}\right)\left(\Theta_{t}, k\right) \chi_{F^{C} \backslash B^{C}}\left(\Theta_{t}\right) d t\right]\right]
$$

which is zero since $\Theta_{\tau_{B}}$ is on the boundary of $B$ a.s. and

$$
E^{\theta}\left[\int_{0}^{\infty} e^{-\lambda t}\left(\Pi_{k}+A \Gamma_{k}-\lambda \Gamma_{k}\right)\left(\Theta_{t}, k\right) \chi_{F^{C} \backslash B^{C}}\left(\Theta_{t}\right) d t\right]=0
$$

for all $\theta \in B$, and also on the boundary of $\mathrm{B}$, since $\mathrm{B}$ is closed. So the result also holds if $\theta \notin B$. 
Lemma 3.7. Let $\mathcal{F}_{q}$ denote $q$-forbidden candidates for all $q \in[0, \infty)$. And suppose that $\mathcal{F}_{q_{2}} \supseteq \mathcal{F}_{q_{1}}$, whenever $q_{2}<q_{1}$. For any $k \in[0, \infty)$, then

$$
\bigcup_{q>k} \mathcal{F}_{q}
$$

and

$$
\bigcap_{q<k} \mathcal{F}_{q}
$$

are both $k$-forbidden candidates.

Proof. To show this first note that if $\theta \in \bigcup_{q>k} \mathcal{F}_{q}$ then since $\left\{\mathcal{F}_{q}\right\}$ is a decreasing family of sets, there exists a $\tilde{q}$ such that $\theta \in \mathcal{F}_{q}$ for all $q \in[k, \tilde{q}]$. This means that

$$
E^{\theta}\left[\int_{0}^{\infty} e^{-\lambda t}\left(\Pi_{k}+A \Gamma_{k}-\lambda \Gamma_{k}\right)\left(\Theta_{t}, q\right) \chi_{\mathcal{F}_{q}^{C}}\left(\Theta_{t}\right) d t\right]=0
$$

for all $q \in[k, \tilde{q}]$, since $\mathcal{F}_{q}$ is a $q$-forbidden candidate. Therefore using the dominated convergence theorem with the function

$$
N^{(\tilde{q})}(\theta)=\sup \left\{\left|\left(\Pi_{k}+A \Gamma_{k}-\lambda \Gamma_{k}\right)(\theta, k)\right|: 0 \leq k \leq \tilde{q}\right\}
$$

from the conditions as a bound, we have

$$
\begin{gathered}
E^{\theta}\left[\int_{0}^{\infty} e^{-\lambda t}\left(\Pi_{k}+A \Gamma_{k}-\lambda \Gamma_{k}\right)\left(\Theta_{t}, k\right) \chi_{\cap_{q>k} \mathcal{F}_{q}^{C}}\left(\Theta_{t}\right) d t\right] \\
=\lim _{q \rightarrow k} E^{\theta}\left[\int_{0}^{\infty} e^{-\lambda t}\left(\Pi_{k}+A \Gamma_{k}-\lambda \Gamma_{k}\right)\left(\Theta_{t}, q\right) \chi_{\mathcal{F}_{q}^{C}}\left(\Theta_{t}\right) d t\right]=0
\end{gathered}
$$

Lemma 3.5 and conditions 2.8.6 then gives that this expression is zero on the boundary also. If $\theta \notin \cup_{q>k} \mathcal{F}_{q}$, then $\theta \notin \mathcal{F}_{q}$ for all $q>k$. So

$$
E^{\theta}\left[\int_{0}^{\infty} e^{-\lambda t}\left(\Pi_{k}+A \Gamma_{k}-\lambda \Gamma_{k}\right)\left(\Theta_{t}, q\right) \chi_{\mathcal{F}_{q}^{C}}\left(\Theta_{t}\right) d t\right] \leq 0
$$

for all $q \geq k$, but then

$$
E^{\theta}\left[\int_{0}^{\infty} e^{-\lambda t}\left(\Pi_{k}+A \Gamma_{k}-\lambda \Gamma_{k}\right)\left(\Theta_{t}, k\right) \chi_{\cap_{q>k} \mathcal{F}_{q}^{C}}\left(\Theta_{t}\right) d t\right] \leq 0
$$

also, since this is the limit as $q \rightarrow k$.

Together this gives that $\bigcup_{q>k} \mathcal{F}_{q}$ is in fact a forbidden candidate. The proof in the other case is similar.

Corollary 3.8. Let $\left\{\mathcal{F}_{k}\right\}_{k \in[0, \infty)}$ be a decreasing family of closed $k$-forbidden candidates, then

$$
\begin{aligned}
& E^{\theta}\left[\int_{0}^{\infty} e^{-\lambda t}\left(\Pi_{k}+A \Gamma_{k}-\lambda \Gamma_{k}\right)\left(\Theta_{t}, k\right) \chi_{\cap_{q>k} \mathcal{F}_{q}^{C}}\left(\Theta_{t}\right) d t\right] \\
= & E^{\theta}\left[\int_{0}^{\infty} e^{-\lambda t}\left(\Pi_{k}+A \Gamma_{k}-\lambda \Gamma_{k}\right)\left(\Theta_{t}, k\right) \chi_{\cup_{q<k} \mathcal{F}_{q}^{C}}\left(\Theta_{t}\right) d t\right] \\
= & E^{\theta}\left[\int_{0}^{\infty} e^{-\lambda t}\left(\Pi_{k}+A \Gamma_{k}-\lambda \Gamma_{k}\right)\left(\Theta_{t}, k\right) \chi_{\mathcal{F}_{k}^{C}}\left(\Theta_{t}\right) d t\right]
\end{aligned}
$$


Proof. Note that $\bigcap_{q<k} \mathcal{F}_{q}$ and $\mathcal{F}_{k}$ are closed and that

$$
\bigcap_{q<k} \mathcal{F}_{q} \supseteq \mathcal{F}_{k} \supseteq \bigcup_{q>k} \mathcal{F}_{q}
$$

Combining lemma 3.6 and lemma 3.7 then gives the result.

Now we are ready to show that the function $\nu(\theta, k)$ is differentiable wrt. $k$.

Lemma 3.9. $\nu(\theta, k)$ is differentiable wrt. $k$ and

$$
\nu_{k}(\theta, k)=E^{\theta}\left[\int_{0}^{\infty} e^{-\lambda t}\left(\Pi_{k}+A \Gamma_{k}-\lambda \Gamma_{k}\right)\left(\Theta_{t}, k\right) \chi_{\mathcal{F}_{k}^{C}}\left(\Theta_{t}\right) d t\right]
$$

Proof. Suppose $q>k$. And let $\Delta k=q-k$. Then we have

$$
\begin{gathered}
\frac{\nu(\theta, q)-\nu(\theta, k)}{\Delta k} \\
=\Delta k^{-1} E^{\theta}\left[\int_{0}^{\infty} e^{-\lambda t}\left[(\Pi+A \Gamma-\lambda \Gamma)\left(\Theta_{t}, q\right)-(\Pi+A \Gamma-\lambda \Gamma)\left(\Theta_{t}, k\right)\right] \chi_{\mathcal{F}_{k}^{C}}\left(\Theta_{t}\right) d t\right] \\
+\Delta k^{-1} E^{\theta}\left[\int_{0}^{\infty} e^{-\lambda t}\left[(\Pi+A \Gamma-\lambda \Gamma)\left(\Theta_{t}, q\right)-(\Pi+A \Gamma-\lambda \Gamma)\left(\Theta_{t}, \phi\left(\Theta_{t}\right)\right)\right] \chi_{\mathcal{F}_{q}^{C} \backslash \mathcal{F}_{k}^{C}}\left(\Theta_{t}\right) d t\right]
\end{gathered}
$$

For the second integral note that whenever $\theta \in \mathcal{F}_{q}^{C} \backslash \mathcal{F}_{k}^{C}$ then $\phi\left(\Theta_{t}\right) \in[k, q]$. Then by the mean value theorem, there exists a function $c(\theta)$ such that $c(\theta) \in[k, q]$ and

$$
\begin{gathered}
\frac{(\Pi+A \Gamma-\lambda \Gamma)\left(\Theta_{t}, q\right)-(\Pi+A \Gamma-\lambda \Gamma)\left(\Theta_{t}, \phi\left(\Theta_{t}\right)\right)}{\Delta k} \chi_{\mathcal{F}_{q}^{C} \backslash \mathcal{F}_{k}^{C}}\left(\Theta_{t}\right) \\
=\left(\Pi_{k}+A \Gamma_{k}-\lambda \Gamma_{k}\right)\left(\Theta_{t}, c\left(\Theta_{t}\right)\right) \chi_{\mathcal{F}_{q}^{C} \backslash \mathcal{F}_{k}^{C}}\left(\Theta_{t}\right) \frac{q-\phi\left(\Theta_{t}\right)}{\Delta k}
\end{gathered}
$$

Note that this function is bounded by the function $N^{(\tilde{k})}\left(\Theta_{t}\right)$ given in the conditions (for some $\tilde{k}>k$ ) and that $\frac{q-\phi\left(\Theta_{t}\right)}{\Delta k} \leq 1$ in $\mathcal{F}_{q}^{C} \backslash \mathcal{F}_{k}^{C}$, so by the dominated convergence theorem, taking the limit from above as $q \rightarrow k$ gives

$$
\begin{gathered}
\lim _{q \rightarrow k} E^{\theta}\left[\frac{(\Pi+A \Gamma-\lambda \Gamma)\left(\Theta_{t}, q\right)-(\Pi+A \Gamma-\lambda \Gamma)\left(\Theta_{t}, \phi\left(\Theta_{t}\right)\right)}{\Delta k} \chi_{\mathcal{F}_{q}^{C} \backslash \mathcal{F}_{k}^{C}}\left(\Theta_{t}\right)\right] \\
\leq E^{\theta}\left[\int_{0}^{\infty} e^{-\lambda t}\left(\Pi_{k}+A \Gamma_{k}-\lambda \Gamma_{k}\right)\left(\Theta_{t}, k\right) \chi_{\cap_{q>k} \mathcal{F}_{q}^{C} \backslash \mathcal{F}_{k}^{C}}\left(\Theta_{t}\right) d t\right]
\end{gathered}
$$

which is zero by corollary 3.8. Then we are left with the first integral

$$
\lim _{q \rightarrow k} \Delta k^{-1} E^{\theta}\left[\int_{0}^{\infty} e^{-\lambda t}\left[(\Pi+A \Gamma-\lambda \Gamma)\left(\Theta_{t}, q\right)-(\Pi+A \Gamma-\lambda \Gamma)\left(\Theta_{t}, k\right)\right] \chi_{\mathcal{F}_{k}^{C}}\left(\Theta_{t}\right) d t\right]
$$

Again using the mean value theorem we can move the limit inside the expectation, since the integrand is bounded by the function $N^{(\tilde{k})}(\theta)$ in the conditions (for some $\tilde{k}>k$ ). This gives

$$
=E^{\theta}\left[\int_{0}^{\infty} e^{-\lambda t}\left(\Pi_{k}+A \Gamma_{k}-\lambda \Gamma_{k}\right)\left(\Theta_{t}, k\right) \chi_{\mathcal{F}_{k}^{C}}\left(\Theta_{t}\right) d t\right]
$$

The case where $q<k$ and $q \rightarrow k$ is similar. 
We have shown that $\nu(\theta, k)$ is differentiable wrt.k and we know what this derivative is.

So the only thing left to prove is that $\nu_{k}(\theta, k)$ satisfies condition 4 in proposition 3.1. This follows quite easily from the definition of the forbidden candidates.

Lemma 3.10.

$$
\nu_{k}(\theta, k) \begin{cases}=0 & \text { for all }(\theta, k) \in \mathcal{F} \\ \leq 0 & \text { for all }(\theta, k) \notin \mathcal{F}\end{cases}
$$

Proof. Fix a $k \in[0, \infty)$. Assume $(\theta, k) \in \mathcal{F}$, that is $\theta \in \mathcal{F}_{k}$. By lemma 3.9 we know that

$$
\nu_{k}(\theta, k)=E^{\theta}\left[\int_{0}^{\infty} e^{-\lambda t}\left(\Pi_{k}+A \Gamma_{k}-\lambda \Gamma_{k}\right)\left(\Theta_{t}, k\right) \chi_{\mathcal{F}_{k}^{C}}\left(\Theta_{t}\right) d t\right]
$$

Now let $\tau$ denote the first hitting time for the process $\Theta_{t}$ to the set $\mathcal{F}_{k}^{C}$. Then

$$
\begin{gathered}
\nu_{k}(\theta, k)=E^{\theta}\left[\int_{0}^{\tau} e^{-\lambda t}\left(\Pi_{k}+A \Gamma_{k}-\lambda \Gamma_{k}\right)\left(\Theta_{t}, k\right) \chi_{\mathcal{F}_{k}^{C}}\left(\Theta_{t}\right) d t\right] \\
+E^{\theta}\left[\int_{\tau}^{\infty} e^{-\lambda t}\left(\Pi_{k}+A \Gamma_{k}-\lambda \Gamma_{k}\right)\left(\Theta_{t}, k\right) \chi_{\mathcal{F}_{k}^{C}}\left(\Theta_{t}\right) d t\right]
\end{gathered}
$$

The first integral is zero, since $\Theta_{t} \in \mathcal{F}_{k}$ as long as $0<t<\tau$. By Dynkin the second integral equals

$$
E^{\theta}\left[e^{-\lambda \tau_{F}} E^{\theta_{\tau_{F}}}\left[\int_{0}^{\infty} e^{-\lambda t}\left(\Pi_{k}+A \Gamma_{k}-\lambda \Gamma_{k}\right)\left(\Theta_{t}, k\right) \chi_{F^{C}}\left(\Theta_{t}\right) d t\right]\right]
$$

This is zero since $\mathrm{F}$ is a forbidden candidate and $\Theta_{\tau_{F}} \in \partial F$.

Now assume that $(\theta, k) \notin \mathcal{F}$. Then $v_{k}(\theta, k) \leq 0$ by the assumption that $\mathcal{F}_{k}$ is a $k$-forbidden candidate.

We have shown that the function defined does in fact satisfy the conditions of proposition 3.1, and therefore it solves the irreversible investment problem. This can be summarized by the following theorem.

Theorem 3.11. Suppose $\Theta_{t}$ is a strong Feller process on the state space $E$ and that the functions $\Pi$ and $\Gamma$ satisfy the conditions 2.8. Given a set $\mathcal{F} \subset E \times[0, \infty)$, and suppose $\mathcal{F}_{k}=\{\theta:(\theta, k) \in \mathcal{F}\}$ satisfies the following:

1. $\mathcal{F}_{k}$ is a $k$-forbidden candidate for every $k$.

2. $\mathcal{F}_{k} \supseteq \mathcal{F}_{q}$ for all $q>k$.

3. If $\theta$ is a boundary point for $\mathcal{F}_{k}$ (except possibly the boundary points of $E$ ), then $\theta \notin \mathcal{F}_{q}$ for any $q>k$.

Let $\phi(\theta)=\sup \{k:(\theta, k) \in \mathcal{F}\}$. And let $\nu(\theta, k)$ be defined by

$$
\begin{gathered}
\nu(\theta, k)=E^{\theta}\left[\int_{0}^{\infty} e^{-\lambda t}(\Pi+A \Gamma-\lambda \Gamma)\left(\Theta_{t}, k\right) \chi_{\mathcal{F}_{k}^{C}}\left(\Theta_{t}\right) d t\right] \\
+E^{\theta}\left[\int_{0}^{\infty} e^{-\lambda t}(\Pi+A \Gamma-\lambda \Gamma)\left(\Theta_{t}, \phi\left(\Theta_{t}\right)\right) \chi_{\mathcal{F}_{k}}\left(\Theta_{t}\right) d t\right]
\end{gathered}
$$


Then

$$
\nu(\theta, k)=\sup \left\{E^{\theta}\left[\int_{0}^{\infty} e^{-\lambda t}(\Pi+A \Gamma-\lambda \Gamma)\left(\Theta_{t}, K_{t}\right) d t\right]: K_{t} \quad(\theta, k) \text {-strategies }\right\}
$$

And the control

$$
K_{t}=k \vee \sup _{s \leq t}\left\{\phi\left(\Theta_{s}\right)\right\}
$$

is an optimal $(\theta, k)$-strategy.

Proof. Lemma 3.4, lemma 3.9 and lemma 3.10 show that the conditions of proposition 3.1 are satisfied.

Proposition 3.12. Suppose B is a Borel set and

$$
E^{\theta}\left[\int_{0}^{\infty} e^{-\lambda t}(\Pi+A \Gamma-\lambda \Gamma)\left(\Theta_{t}, k\right) \chi_{B}\left(\Theta_{t}\right) d t\right]
$$

is continuous in $\theta$ for all $k$, then the result in theorem 3.11 holds for all strongly measurable strong Markov processes and $\left(\Pi_{k}+A \Gamma_{k}-\lambda \Gamma_{k}\right)(\theta, k)$ not necessarily bounded.

Proof. The boundedness and the strong Feller properties are only needed to show the $\theta$ continuity of the function above (which is only needed in lemma 3.7. Then the result follows as in theorem 3.11 


\section{Diffusions}

Suppose $\Theta_{t}$ is a one dimensional diffusion on the state space $(0, \infty)$, where the drift $\mu(\theta)$ and variance $\sigma^{2}(\theta)$ both are continuous on $(0, \infty)$ and

$$
\sigma^{2}(\theta)>0 \text { for all } \theta \in(0, \infty)
$$

then it is possible to show that the function given in proposition 3.12 is continuous as long as $\left(\Pi_{k}+A \Gamma_{k}-\lambda \Gamma_{k}\right)(\theta, k) \in L_{\Theta}^{1}$. Then will not need to show that diffusions satisfying the properties above are strong Feller processes.

\subsection{Resolvent of a diffusion}

As seen in the previous sections the resolvent plays an important part in finding the solution of the irreversible investment problem. In order to get more explicit solutions, it is useful to use an alternative expression for the resolvent. As we shall se this expression is equal to the resolvent for all the functions we will work with i.e. the functions in $L_{\Theta}^{1}$. But first a result from Breiman [2] is useful. It gives the existence of two independent solutions of the equation $A f-\lambda f=0$ that are easy to work with.

Theorem 4.1 (Breiman [2], Theorem 16.69). $A \phi=\lambda \phi, \lambda>0$, has two continuous solutions $\phi_{+}(\theta), \phi_{-}(\theta)$ such that

1. $\phi_{+}(\theta)>0, \phi_{-}(\theta)>0, \theta \in(0, \infty)$

2. $\phi_{+}(\theta)$ is strictly increasing, $\phi_{-}(\theta)$ is strictly decreasing.

Proof. See Breiman [2] pg.380 for the proof.

It can be shown that if the process is a diffusion, then the functions $\phi_{+}$and $\phi_{-}$satisfy the corresponding differential equation

$$
\frac{1}{2} \sigma^{2}(\theta) \phi^{\prime \prime}(\theta)+\mu(\theta) \phi^{\prime}-\lambda \phi=0
$$

(See Breiman [2] pg.389). In the following definition $W(\theta)$ denotes the wronskian of the functions $\phi_{+}(\theta)$ and $\phi_{-}(\theta)$, so

$$
W(\theta)=\phi_{+}^{\prime}(\theta) \phi_{-}(\theta)-\phi_{+}(\theta) \phi_{-}^{\prime}(\theta)
$$

Since $\phi_{+}$and $\phi_{-}$are twice differentiable it follows that the wronskian is continuous in $(0, \infty)$. In addition it is also strictly positive using Breimans theorem. Using any functions $\phi_{+}(\theta)$ and $\phi_{-}(\theta)$ that satisfy the properties in theorem 4.1 we have the following definition.

Definintion 4.2. Let $L_{\phi_{+}, \phi_{-}}^{1}$ be the set of measurable functions such that

$$
\phi_{-}(\theta) \int_{0}^{\theta} \frac{\phi_{+}(\eta)|f(\eta)|}{W(\eta) \sigma^{2}(\eta)} d \eta
$$

and

$$
\phi_{+}(\theta) \int_{\theta}^{\infty} \frac{\phi_{-}(\eta)|f(\eta)|}{W(\eta) \sigma^{2}(\eta)} d \eta
$$

are bounded for $\theta \in(0, \infty)$. 
The functions in $L_{\phi_{+}, \phi_{-}}^{1}$ are interesting because the resolvent of such functions can be expressed using the functions $\phi_{+}(\theta)$ and $\phi_{-}(\theta)$.

Proposition 4.3. Suppose $f \in L_{\phi_{+}, \phi_{-}}^{1}$. Then

$$
R_{\lambda} f(\theta)=\phi_{-}(\theta) \int_{0}^{\theta} \frac{2 \phi_{+}(\eta) f(\eta)}{W(\eta) \sigma^{2}(\eta)} d \eta+\phi_{+}(\theta) \int_{\theta}^{\infty} \frac{2 \phi_{-}(\eta) f(\eta)}{W(\eta) \sigma^{2}(\eta)} d \eta
$$

Proof. Let

$$
u(\theta)=\phi_{-}(\theta) \int_{0}^{\theta} \frac{2 \phi_{+}(\eta) f(\eta)}{W(\eta) \sigma^{2}(\eta)} d \eta+\phi_{+}(\theta) \int_{\theta}^{\infty} \frac{2 \phi_{-}(\eta) f(\eta)}{W(\eta) \sigma^{2}(\eta)} d \eta
$$

First assume that $f$ is continuous and in $L_{\phi_{+}, \phi_{-}}^{1}$. Then $u(\theta)$ satisfies the differential equation

$$
A u-\lambda u=-f
$$

By theorem 2.3 this equation has only one bounded solution in $\mathcal{D}_{A}$, and this solution is the resolvent of $f$. Therefore $u(\theta)=R_{\lambda} f(\theta)$. The result therefore holds if the function $f$ is continuous and in $L_{\phi_{+}, \phi_{-}}^{1}$. By using the convergence theorems the result can be seen to hold for all functions in $L_{\phi_{+}, \phi_{-}}^{1}$.

Naturally one wants to ask which functions are in fact in $L_{\phi_{+}, \phi_{-}}^{1}$. The following proposition gives the connection between the previously defined $L_{\Theta}^{1}$ and $L_{\phi_{+}, \phi_{-}}^{1}$.

Proposition 4.4.

$$
L_{\Theta}^{1} \subseteq L_{\phi_{+}, \phi_{-}}^{1}
$$

Proof. First assume that $f$ is a bounded function. Then $E^{\theta}\left[e^{-\lambda t}\left|f\left(\Theta_{t}\right)\right| d t\right]$ is bounded for $\theta \in(0, \infty)$. Let

$$
f_{n}(\theta)=\min \left\{n,|f(\theta)| \chi_{\left[\frac{1}{n}, n\right]}(\theta)\right\}
$$

Note now that the functions $\phi_{+}(\theta), \phi_{-}(\theta), \sigma^{2}(\theta)$ and $W(\theta)$ are continuous so they are bounded on the interval $\left[\frac{1}{n}, n\right]$ for all $n$. In addition $f_{n}$ is bounded for every $n$. Then the integral

$$
\int_{0}^{\theta} \frac{2 \phi_{+}(\eta) f_{n}(\eta)}{W(\eta) \sigma^{2}(\eta)} d \eta
$$

is an integral of a bounded function over a bounded interval, so the integral is bounded for all $\theta . \phi_{-}(\theta)$ is bounded in $\left[\frac{1}{n}, n\right]$ (and in $\left[\frac{1}{n}, \infty\right)$ since it is decreasing). Also whenever $\theta<\frac{1}{n}$, then the integral is zero, so

$$
\phi_{-}(\theta) \int_{0}^{\theta} \frac{2 \phi_{+}(\eta) f_{n}(\eta)}{W(\eta) \sigma^{2}(\eta)} d \eta
$$

is bounded for $\theta \in(0, \infty)$. In a similar way

$$
\phi_{+}(\theta) \int_{\theta}^{\infty} \frac{2 \phi_{-}(\eta) f_{n}(\eta)}{W(\eta) \sigma^{2}(\eta)} d \eta
$$


is also bounded. Then by proposition 4.3 we have that for all $n$

$$
E^{\theta}\left[\int_{0}^{\infty} e^{-\lambda t} f_{n}\left(\Theta_{t}\right) d t\right]=\phi_{-}(\theta) \int_{0}^{\theta} \frac{2 \phi_{+}(\eta) f_{n}(\eta)}{W(\eta) \sigma^{2}(\eta)} d \eta+\phi_{+}(\theta) \int_{\theta}^{\infty} \frac{2 \phi_{-}(\eta) f_{n}(\eta)}{W(\eta) \sigma^{2}(\eta)} d \eta
$$

Now $f_{n}(\theta) \leq|f(\theta)|$, so therefore

$$
E^{\theta}\left[\int_{0}^{\infty} e^{-\lambda t} f_{n}\left(\Theta_{t}\right) d t\right] \leq E^{\theta}\left[\int_{0}^{\infty} e^{-\lambda t}\left|f\left(\Theta_{t}\right)\right| d t\right]
$$

But $E^{\theta}\left[\int_{0}^{\infty} e^{-\lambda t}\left|f\left(\Theta_{t}\right)\right| d t\right]$ is bounded by a constant M. Then

$$
\phi_{-}(\theta) \int_{0}^{\theta} \frac{2 \phi_{+}(\eta) f_{n}(\eta)}{W(\eta) \sigma^{2}(\eta)} d \eta+\phi_{+}(\theta) \int_{\theta}^{\infty} \frac{2 \phi_{-}(\eta) f_{n}(\eta)}{W(\eta) \sigma^{2}(\eta)} d \eta
$$

is also bounded by $M$ for $\theta \in(0, \infty)$ for all $n$. Since $\frac{2 \phi_{-}(\eta) f_{n}(\eta)}{W(\eta) \sigma^{2}(\eta)}$ is positive and increasing in $n$ for every $\eta$, the monotone convergence theorem gives

$$
\begin{gathered}
\phi_{-}(\theta) \int_{0}^{\theta} \frac{2 \phi_{+}(\eta)|f(\eta)|}{W(\eta) \sigma^{2}(\eta)} d \eta+\phi_{+}(\theta) \int_{0}^{\infty} \frac{2 \phi_{-}(\eta)|f(\eta)|}{W(\eta) \sigma^{2}(\eta)} d \eta \\
=\lim _{n \rightarrow \infty} \phi_{-}(\theta) \int_{0}^{\theta} \frac{\phi_{+}(\eta) f_{n}(\eta)}{W(\eta) \sigma^{2}(\eta)} d \eta+\lim _{n \rightarrow \infty} \phi_{+}(\theta) \int_{\theta}^{\infty} \frac{f_{n}(\eta) \phi_{-}(\eta)}{W(\eta) \sigma^{2}(\eta)} d \eta \leq M
\end{gathered}
$$

Then $f \in L_{\phi_{+}, \phi_{-}}^{1}$.

This gives the following observation.

Corollary 4.5. If $f \in L_{\Theta}^{1}$, then

$$
R_{\lambda} f(\theta)=\phi_{-}(\theta) \int_{0}^{\theta} \frac{2 \phi_{+}(\eta) f(\eta)}{W(\eta) \sigma^{2}(\eta)} d \eta+\phi_{+}(\theta) \int_{\theta}^{\infty} \frac{2 \phi_{-}(\eta) f(\eta)}{W(\eta) \sigma^{2}(\eta)} d \eta
$$

Proof. Since $L_{\Theta}^{1} \subseteq L_{\phi_{+}, \phi_{-}}^{1}$, then the result follows by proposition 4.3.

This result is useful because it gives an explicit expression for the resolvent of measurable functions, but also because it gives a sufficient condition and also a nescessary condition for a function to be in $L_{\Theta}^{1}$. In fact showing directly that the bounded functions are in $L_{\phi_{+}, \phi_{-}}^{1}$ requires some of work, especially if the functions $\phi_{+}(\theta)$ and $\phi_{-}(\theta)$ are not given explicitly. But knowing that $L_{\phi_{+}, \phi_{-}}^{1}=L_{\Theta}^{1}$ this is obviously the case.

\subsection{A simplified problem}

In this section we study a simplified problem. Here we will prove the existence of a solution and also give an explicit formula for the solution. We will assume that the set

$$
I_{k}^{C}=\left\{\theta:\left(\Pi_{k}+A \Gamma_{k}-\lambda \Gamma_{k}\right)(\theta, k)<0\right\}
$$

is an interval for every $k$. Then $I_{k}^{C}$ is of the form $(0, a) \cup(b, \infty)$ where one or both of these intervals possibly are empty sets. So let us assume that the $k$-forbidden candidate is of the form $\left[\psi_{1}, \psi_{2}\right]$. Then we want to find numbers $\psi_{1}$ and $\psi_{2}$ such that

$$
E^{\theta}\left[\int_{0}^{\infty} e^{-\lambda t}\left(\Pi_{k}+A \Gamma_{k}-\lambda \Gamma_{k}\right)\left(\Theta_{t}, k\right) \chi_{\left(0, \psi_{1}\right)}\left(\Theta_{t}\right) d t\right]
$$




$$
+E^{\theta}\left[\int_{0}^{\infty} e^{-\lambda t}\left(\Pi_{k}+A \Gamma_{k}-\lambda \Gamma_{k}\right)\left(\Theta_{t}, k\right) \chi_{\left(\psi_{2}, \infty\right)}\left(\Theta_{t}\right) d t\right]=0
$$

for $\theta=\psi_{1}$ and $\theta=\psi_{2}$. Lets first try to evaluate this function for $\theta=\psi_{1}$. Then using the alternative expression for the resolvent given in the previous section, this equals

$$
\phi_{-}\left(\psi_{1}\right) \int_{0}^{\psi_{1}} \frac{2 \phi_{+}(\eta)\left(\Pi_{k}+A \Gamma_{k}-\lambda \Gamma_{k}\right)(\eta, k)}{W(\eta) \sigma^{2}(\eta)} d \eta+\phi_{+}\left(\psi_{1}\right) \int_{\psi_{2}}^{\infty} \frac{2 \phi_{-}(\eta)\left(\Pi_{k}+A \Gamma_{k}-\lambda \Gamma_{k}\right)(\eta, k)}{W(\eta) \sigma^{2}(\eta)} d \eta
$$

We need to choose $\psi_{1}$ and $\psi_{2}$ such that these integrals equal zero, and at the same time ensuring that the interval $\left(0, \psi_{1}\right)$ contains the interval $(0, a)$. This suggests that we let $\psi_{1}$ and $\psi_{2}$ be defined as follows:

$$
\psi_{1}=\inf \left\{\theta \in(0, \infty): \int_{0}^{\theta} \frac{\phi_{+}(\eta)\left(\Pi_{k}+A \Gamma_{k}-\lambda \Gamma_{k}\right)(\eta, k)}{W(\eta) \sigma^{2}(\eta)} d \eta>0\right\}
$$

and

$$
\psi_{2}=\sup \left\{\theta \in(0, \infty): \int_{\theta}^{\infty} \frac{\phi_{-}(\eta)\left(\Pi_{k}+A \Gamma_{k}-\lambda \Gamma_{k}\right)(\eta, k)}{W(\eta) \sigma^{2}(\eta)} d \eta>0\right\}
$$

The following two lemmas verify that the given assumptions are in fact correct. A similar result can be found in the Kobila article [7].

Lemma 4.6. Let $f$ be a continuous function in $L_{\Theta}^{1}$ such that

$$
\int_{\theta}^{a} f(\eta) d \eta \geq 0, \text { for all } \theta \in(0, a)
$$

Then

$$
\int_{\theta}^{a} f(\eta)\left(1-\left(\frac{\phi_{+}(\theta) \phi_{-}(\eta)}{\phi_{-}(\theta) \phi_{+}(\eta)}\right)\right) d \eta \geq 0, \text { for all } \theta \in(0, a)
$$

Proof. Define

$$
g(\theta)=\frac{\phi_{-}(\theta)}{\phi_{+}(\theta)} \int_{\theta}^{a} f(\eta) d \eta-\int_{\theta}^{a} \frac{\phi_{-}(\eta)}{\phi_{+}(\eta)} f(\eta) d \eta
$$

Note now that $g(a)=0$ and that the function $g(\theta)$ is differentiable.

$$
\begin{gathered}
g^{\prime}(\theta)=\frac{\phi_{-}^{\prime}(\theta) \phi_{+}(\theta)-\phi_{+}^{\prime}(\theta) \phi_{-}(\theta)}{\left(\phi_{+}(\theta)\right)^{2}} \int_{\theta}^{a} f(\eta) d \eta-\frac{\phi_{-}(\theta)}{\phi_{+}(\theta)} f(\theta)+\frac{\phi_{-}(\theta)}{\phi_{+}(\theta)} f(\theta) \\
=\frac{\phi_{-}^{\prime}(\theta) \phi_{+}(\theta)-\phi_{+}^{\prime}(\theta) \phi_{-}(\theta)}{\left(\phi_{+}(\theta)\right)^{2}} \int_{\theta}^{a} f(\eta) d \eta
\end{gathered}
$$

The expression $\phi_{+}^{\prime}(\theta) \phi_{-}(\theta)-\phi_{-}^{\prime} \phi_{+}(\theta)$ is positive since $\phi_{+}(\theta)$ is strictly increasing and $\phi_{-}(\theta)$ is strictly decreasing. Using the assumption that $\int_{\theta}^{a} f(\eta) d \eta \geq 0$, gives that $g(\theta)$ is in fact a monotonely decreasing function, and since $g(a)=0$, then $g(\theta) \geq 0$ for all $\theta \in(0, a)$. This also gives that the integral in question is positive by considering $\frac{\phi_{+}(\theta)}{\phi_{-}(\theta)} g(\theta)$.

Functions $\psi_{1}(k)$ and $\psi_{2}(k)$ can be defined in a similar way as in Kobilas article such that the interval $\left[\psi_{1}(k), \psi_{2}(k)\right]$ is in fact a $k$-forbidden candidate for every $k$. 
Lemma 4.7. Let the functions $\psi_{1}(k)$ and $\psi_{2}(k)$ be defined by

$$
\begin{gathered}
\psi_{1}(k)=\inf \left\{\theta>0: \int_{0}^{\theta} \frac{\phi_{+}(\eta)\left(\Pi_{k}+A \Gamma_{k}-\lambda \Gamma_{k}\right)(\eta, k)}{W(\eta) \sigma^{2}(\eta)} d \eta>0\right\} \\
\psi_{2}(k)=\sup \left\{\theta>0: \int_{\theta}^{\infty} \frac{\phi_{-}(\eta)\left(\Pi_{k}+A \Gamma_{k}-\lambda \Gamma_{k}\right)(\eta, k)}{W(\eta) \sigma^{2}(\eta)} d \eta>0\right\}
\end{gathered}
$$

Let $\mathcal{F}_{k}=\left\{\theta: \psi_{1}(k) \leq \theta \leq \psi_{2}(k)\right\}$. Then $\mathcal{F}_{k}$ is a $k$-forbidden candidate for every $k \in[0, \infty)$.

Proof. Fix a $k \in[0, \infty)$. Note that by corollary 4.5 then

$$
\begin{gathered}
E^{\theta}\left[\int_{0}^{\infty} e^{-\lambda t}\left(\Pi_{k}+A \Gamma_{k}-\lambda \Gamma_{k}\right)\left(\Theta_{t}, k\right) \chi_{\mathcal{F}_{k}^{C}}\left(\Theta_{t}\right) d t\right] \\
=\phi_{-}(\theta) \int_{0}^{\theta} \frac{2 \phi_{+}(\eta)\left(\Pi_{k}+A \Gamma_{k}-\lambda \Gamma_{k}\right)(\eta, k) \chi_{\left[\psi_{1}(k), \psi_{2}(k)\right]^{C}}(\eta)}{W(\eta) \sigma^{2}(\eta)} d \eta \\
+\phi_{+}(\theta) \int_{\theta}^{\infty} \frac{2 \phi_{-}(\eta)\left(\Pi_{k}+A \Gamma_{k}-\lambda \Gamma_{k}\right)(\eta, k) \chi_{\left[\psi_{1}(k), \psi_{2}(k)\right]^{C}}(\eta)}{W(\eta) \sigma^{2}(\eta)} d \eta
\end{gathered}
$$

If $\psi_{1}(k) \leq \psi_{2}(k)$, inserting $\theta=\psi_{1}(k)$ gives:

$$
\begin{aligned}
& =\phi_{-}\left(\psi_{1}(k)\right) \int_{0}^{\psi_{1}(k)} \frac{2 \phi_{+}(\eta)\left(\Pi_{k}+A \Gamma_{k}-\lambda \Gamma_{k}\right)(\eta, k) \chi_{\left(0, \psi_{1}(k)\right)}(\eta)}{W(\eta) \sigma^{2}(\eta)} d \eta \\
& +\phi_{+}\left(\psi_{1}(k)\right) \int_{\psi_{2}(k)}^{\infty} \frac{2 \phi_{-}(\eta)\left(\Pi_{k}+A \Gamma_{k}-\lambda \Gamma_{k}\right)(\eta, k) \chi_{\left(\psi_{2}(k), \infty\right)}(\eta)}{W(\eta) \sigma^{2}(\eta)} d \eta
\end{aligned}
$$

these integrals are zero by the definition of $\psi_{1}(k)$ and $\psi_{2}(k)$. The result is similar for $\theta=\psi_{2}(k)$. It remains to show that the set $\mathcal{F}_{k}$ also satisfies the last two properties of forbidden candidates.

Assume $\theta \in\left(0, \psi_{1}(k)\right)$. Then

$$
\begin{gathered}
E^{\theta}\left[\int_{0}^{\infty} e^{-\lambda t}\left(\Pi_{k}+A \Gamma_{k}-\lambda \Gamma_{k}\right)\left(\Theta_{t}, k\right) \chi_{\left[\psi_{1}(k), \psi_{2}(k)\right]^{C}}\left(\Theta_{t}\right) d t\right] \\
=\phi_{-}(\theta) \int_{0}^{\theta} \frac{2 \phi_{+}(\eta)\left(\Pi_{k}+A \Gamma_{k}-\lambda \Gamma_{k}\right)(\eta, k)}{W(\eta) \sigma^{2}(\eta)} d \eta+\phi_{+}(\theta) \int_{\theta}^{\psi_{1}(k)} \frac{2 \phi_{-}(\eta)\left(\Pi_{k}+A \Gamma_{k}-\lambda \Gamma_{k}\right)(\eta, k)}{W(\eta) \sigma^{2}(\eta)} d \eta \\
+\phi_{+}(\theta) \int_{\psi_{2}(k)}^{\infty} \frac{2 \phi_{-}(\eta)\left(\Pi_{k}+A \Gamma_{k}-\lambda \Gamma_{k}\right)(\eta, k)}{W(\eta) \sigma^{2}(\eta)} d \eta
\end{gathered}
$$

Note that the last of these integrals is zero, and since

$$
\phi_{-}(\theta) \int_{0}^{\psi_{1}(k)} \frac{2 \phi_{+}(\eta)\left(\Pi_{k}+A \Gamma_{k}-\lambda \Gamma_{k}\right)(\eta, k)}{W(\eta) \sigma^{2}(\eta)} d \eta=0
$$


the first integral in $\left(^{*}\right)$ can be rewritten. This gives

$$
\begin{gathered}
=\phi_{+}(\theta) \int_{\theta}^{\psi_{1}(k)} \frac{2 \phi_{-}(\eta)\left(\Pi_{k}+A \Gamma_{k}-\lambda \Gamma_{k}\right)(\eta, k)}{W(\eta) \sigma^{2}(\eta)} d \eta-\phi_{-}(\theta) \int_{\theta}^{\psi_{1}(k)} \frac{2 \phi_{+}(\eta)\left(\Pi_{k}+A \Gamma_{k}-\lambda \Gamma_{k}\right)(\eta, k)}{W(\eta) \sigma^{2}(\eta)} d \eta \\
=-\phi_{-}(\theta) \int_{\theta}^{\psi_{1}(k)} \frac{2 \phi_{+}(\eta)\left(\Pi_{k}+A \Gamma_{k}-\lambda \Gamma_{k}\right)(\eta, k)}{W(\eta) \sigma^{2}(\eta)}\left(1-\frac{\phi_{+}(\theta) \phi_{-}(\eta)}{\phi_{-}(\theta) \phi_{+}(\eta)}\right) d \eta
\end{gathered}
$$

which is negative for all $\theta \in(0, \psi(k))$ by lemma 4.6 .

If $U_{k}^{C}$ is connected, then $U_{k}$ can be written as a union of two intervals $(0, a)$ and $(b, \infty)$ (where one or both of these intervals possibly are empty sets). It is easily seen that $\psi_{1}(k)>a$ and $\psi_{2}(k)<b$. This means that $\left[\psi_{1}(k), \psi_{2}(k)\right]$ is a $k$-forbidden candidate for each $k$.

It remains to show the monotonicity of the family of forbidden candidates. More precisely we need to show the following

Lemma 4.8. If $q>k$ then $\left[\psi_{1}(k), \psi_{2}(k)\right] \supseteq\left[\psi_{1}(q), \psi_{2}(q)\right]$

Proof. Assume that $\psi_{1}(k)>0$. By definition

$$
\int_{0}^{\psi_{1}(k)} \frac{\phi_{+}(\eta)\left(\Pi_{k}+A \Gamma_{k}-\lambda \Gamma_{k}\right)(\eta, k)}{W(\eta) \sigma^{2}(\eta)} d \eta=0
$$

Since $\left(\Pi_{k}+A \Gamma_{k}-\lambda \Gamma_{k}\right)(\theta, k)$ is strictly decreasing in the $k$-variable, then

$$
\int_{0}^{\psi_{1}(k)} \frac{\phi_{+}(\eta)\left(\Pi_{k}+A \Gamma_{k}-\lambda \Gamma_{k}\right)(\eta, q)}{W(\eta) \sigma^{2}(\eta)} d \eta<0
$$

for all $q>k$. For all such $q$ there exists a $\tilde{\theta}>\psi_{1}(k)$ such that

$$
\int_{0}^{\tilde{\theta}} \frac{\phi_{+}(\eta)\left(\Pi_{k}+A \Gamma_{k}-\lambda \Gamma_{k}\right)(\eta, q)}{W(\eta) \sigma^{2}(\eta)} d \eta<0
$$

also. Then $\psi_{1}(q)>\tilde{\theta}$, so the function $\psi_{1}(k)$ is strictly increasing. Similar arguments also give that $\psi_{2}(k)$ is strictly decreasing. The result then follows.

Note that since $\psi_{1}(k)$ and $\psi_{2}(k)$ are the only boundary points (except 0 and $\infty$ ), and $\psi_{1}(k)$ is strictly increasing and $\psi_{2}(k)$ is strictly decreasing, then the boundary points of $\left[\psi_{1}(k), \psi_{2}(k)\right]$ are not in $\left[\psi_{1}(q), \psi_{2}(q)\right]$ for any $q>k$. The solution to the optimal irreversible investment problem can be summarized in the following theorem:

Theorem 4.9. Suppose $\Pi$ and $\Gamma$ are functions satisfying the conditions in 2.8.1-5. Let

$$
\begin{gathered}
\psi_{1}(k)=\inf \left\{\theta \in(0, \infty): \int_{0}^{\theta} \frac{\phi_{+}(\eta)\left(\Pi_{k}+A \Gamma_{k}-\lambda \Gamma_{k}\right)(\eta, k)}{W(\eta) \sigma^{2}(\eta)} d \eta>0\right\} \\
\psi_{2}(k)=\sup \left\{\theta \in(0, \infty): \int_{\theta}^{\infty} \frac{\phi_{-}(\eta)\left(\Pi_{k}+A \Gamma_{k}-\lambda \Gamma_{k}\right)(\eta, k)}{W(\eta) \sigma^{2}(\eta)} d \eta>0\right\}
\end{gathered}
$$


Let $\mathcal{F}=\left\{(\theta, k): \psi_{1}(k) \leq \theta \leq \psi_{2}(k)\right\}$ and $\phi(\theta)=\sup \{k:(\theta, k) \in \mathcal{F}\}$. Let the function $h(\eta, k)$ be defined by

$$
h(\eta, k)= \begin{cases}(\eta, k) & \text { for } \eta \notin\left[\psi_{1}(k), \psi_{2}(k)\right] \\ (\eta, \phi(\eta)) & \text { for } \eta \in\left[\psi_{1}(k), \psi_{2}(k)\right]\end{cases}
$$

Then

$$
\begin{gathered}
\sup \left\{E^{(\theta, k)}\left[\int_{0}^{\infty} e^{-\lambda t}(\Pi+A \Gamma-\lambda \Gamma)\left(\Theta_{t}, K_{t}\right) d t\right]:(\theta, k) \text {-strategies }\right\} \\
=\phi_{-}(\theta) \int_{0}^{\theta} \frac{2 \phi_{+}(\eta)(\Pi+A \Gamma-\lambda \Gamma)(h(\eta, k))}{W(\eta) \sigma^{2}(\eta)} d \eta+\phi_{+}(\theta) \int_{\theta}^{\infty} \frac{2 \phi_{-}(\eta)(\Pi+A \Gamma-\lambda \Gamma)(h(\eta, k))}{W(\eta) \sigma^{2}(\eta)} d \eta
\end{gathered}
$$

And the optimal control is

$$
K_{t}=k \vee \sup \left\{\phi\left(\theta_{s}\right): s \leq t\right\}
$$

Proof. Using lemma 4.7, lemma 4.8, with the following remark, then proposition 3.12 gives the result.

Let us end this section with an example.

Example 4.1. Suppose we have the market process

$$
d \Theta_{t}=\frac{1}{\Theta_{t}} d t+d B_{t}
$$

with discount factor $\lambda=\frac{1}{2}$. Let $\Pi$ and $\Gamma$ be the functions

$$
\begin{aligned}
\Pi(\theta, k) & =\frac{1}{\theta}\left(1-\frac{1}{k+1}\right) \\
\Gamma(\theta, k) & =k
\end{aligned}
$$

Then

$$
(\Pi+A \Gamma-\lambda \Gamma)(\theta, k)=\frac{1}{\theta}\left(1-\frac{1}{k+1}\right)-\frac{1}{2} k
$$

and

$$
\left(\Pi_{k}+A \Gamma_{k}-\lambda \Gamma_{k}\right)(\theta, k)=\frac{1}{\theta(k+1)^{2}}-\frac{1}{2}
$$

Note that the equation $A \phi-\lambda \phi=0$ has the two solutions

$$
\phi_{+}(\theta)=\frac{e^{\theta}-e^{-\theta}}{\theta} \quad \text { and } \quad \phi_{-}(\theta)=\frac{e^{-\theta}}{\theta}
$$

and that these are on form as in proposition 4.3. This gives $W(\theta)=\frac{2}{\theta^{2}}$.

$$
S^{+}(\theta)=\max \left\{0, \sup _{k \geq 0}\{(\Pi+A \Gamma-\lambda \Gamma)(\theta, k)\}\right\} \leq \frac{1}{\theta}
$$


which is in $L_{\Theta}^{1}$ by proposition 4.4. Also

$$
\begin{gathered}
M^{(q)}(\theta)=\sup \{|(\Pi+A \Gamma-\lambda \Gamma)(\theta, k)|: k \in[0, q]\} \leq \frac{1}{\theta}+\lambda q \\
N^{(q)}(\theta)=\sup \left\{\left|\left(\Pi_{k}+A \Gamma_{k}-\lambda \Gamma_{k}\right)(\theta, k)\right|: k \in[0, q]\right\} \leq \frac{1}{\theta}+\lambda
\end{gathered}
$$

which are in $L_{\Theta}^{1}$ for all $q \in[0, \infty) .\left(\Pi_{k}+A \Gamma_{k}-\lambda \Gamma_{k}\right)(\theta, k)$ is strictly decreasing in the $k$-variable, since

$$
\left(\Pi_{k k}+A \Gamma_{k k}-\lambda \Gamma_{k k}\right)(\theta, k)=-\frac{1}{\theta(k+1)^{3}}
$$

which clearly is negative for all $(\theta, k) \in(0, \infty) \times[0, \infty)$. So all the conditions on the functions $\Pi$ and $\Gamma$ are satisfied. Note that for all $k$ there exists a $\delta>0$ such that $\left(\Pi_{k}+\right.$ $\left.A \Gamma_{k}-\lambda \Gamma_{k}\right)(\theta, k)>0$ for all $\theta \in(0, \delta)$. Then $\psi_{1}(k)=0$ for all $k$. By proposition 4.4 the function $\psi_{2}(k)$ becomes

$$
\psi_{2}(k)=\sup \left\{\theta \in(0, \infty): \int_{\theta}^{\infty} \frac{1}{(k+1)^{2}} e^{-\eta}-\frac{1}{2} \eta e^{-\eta} d \eta\right\}
$$

The integral can be computed quite easily

$$
\int_{\theta}^{\infty} \frac{1}{(k+1)^{2}} e^{-\eta}-\frac{1}{2} \eta e^{-\eta} d \eta=\frac{1}{(k+1)^{2}} e^{-\theta}-\frac{1}{2} \theta e^{-\theta}-\frac{1}{2} e^{-\theta}=\left(\frac{1}{(k+1)^{2}}-\frac{1+\theta}{2}\right) e^{-\theta}
$$

For each $k$ there exists only one point $\theta$ for which the expression is zero, and this point is

$$
\theta=\frac{2}{(k+1)^{2}}-1
$$

So therefore $\psi_{2}(k)=\max \left\{\frac{2}{(k+1)^{2}}-1,0\right\}$. And the forbidden region that solves the irreversible investment problem is

$$
\mathcal{F}=\left\{(\theta, k): 0<\theta<\frac{2}{(k+1)^{2}}-1\right\}
$$




\section{References}

[1] Halvard Arntzen (1994) : Solution to a class of stochastic investment problems involving finite variation controls. Preprint series, University of Oslo.

[2] Leo Breimann (1968) : Probability. Addison-Wesley publishing company

[3] Avinash Dixit and Robert Pindyck (1994) : Investment under uncertainty. Princeton university press.

[4] E.B.Dynkin (1965) : Markov processes - volume I. Springer-Verlag.

[5] E.B.Dynkin (1965) : Markov processes - volume II. Springer-Verlag.

[6] Inge Helland (1996) : One-dimensional diffusion processes and their boundaries. Statistical research report, university of Oslo.

[7] T.Ø.Kobila (1993) : A Class of Solvable Stochastic Investment Problems Involving Singular Controls. Stochastics and stochastic reports 43, pg.2963.

[8] Philip Protter (1990) : Stochastic integration and differential equations. Springer-Verlag.

[9] Anders Øksendal (1997) : Irreversible investment problems. Cand. Scient. thesis, University of Oslo.

[10] Bernt Øksendal (1995) : Stochastic differential equations. Springer-Verlag. 
